13. J.Y. Kim and G.Y. Lee, Wideband and compact bandstop filter structure using double-plane superposition, IEEE Microwave Wireless Compon Lett 8 (1998), 69-71.

14. T. Akalin, M. Laso, E. Delos, T. Lopetegi, O. Vanbesien, M. Sorolla, and D. Lippens, High performance double-sided microstrip PBG filter, Microwave Opt Technol Lett 13 (2003), 279-280.

15. C. Balanis, Antenna theory analysis and design, Wiley, New York, 1997.

(C) 2005 Wiley Periodicals, Inc.

\section{SPECTRALLY ACCELERATED BICONJUGATE GRADIENT STABILIZED METHOD FOR SCATTERING FROM AND PROPAGATION OVER ELECTRICALLY LARGE INHOMOGENEOUS GEOMETRIES}

Baris Babaoglu, Ayhan Altintas, and Vakur B. Ertürk

Department of Electrical and Electronics Engineering

Bilkent University

TR-06800, Bilkent Ankara, Turkey

Received 4 January 2005

ABSTRACT: Scattering from and propagation over rough-terrain profiles, as well as reentrant surfaces are investigated using an integral equation (IE)-based spectrally accelerated biconjugate gradient stabilized (SA-BiCGSTAB) method, with a storage requirement and a computational cost of $O(N)$ per iteration, where $N$ is the surface unknowns in the discretized IE. Numerical results in the form of current and path loss are presented and compared with previously published as well as measured results in order to assess the accuracy and efficiency of this method. (C) 2005 Wiley Periodicals, Inc. Microwave Opt Technol Lett 46: 158-162, 2005; Published online in Wiley InterScience (www. interscience.wiley.com). DOI 10.1002/mop.20930

Key words: biconjugate gradient stabilized method; electromagnetic rough-surface scattering; method of moments; spectral acceleration; propagation over terrain

\section{INTRODUCTION}

Accurate analysis of electromagnetic-field strengths and propagation over rough-surface profiles has great importance for military and commercial applications such as frequency-channel planning, coverage area estimation, and so forth. Therefore, various solution methods have been proposed. Majority of them are propagationprediction models such as the Okumura, Hata, and ITU-370 approaches [1-3], which depend on empirical formulas obtained via statistical analysis, yielding general scattering or diffraction properties. More accurate predictions can be obtained using integral equation (IE)-based methods solved via the method of moments (MoM). However, they suffer from the storage and the computational-cost requirements when applied to electrically large geometries, even though using an iterative method can alleviate the computational cost to some extent. Therefore, the spectral acceleration (SA) algorithm was developed in [4] for slightly rough surfaces and modified in [5] to handle very undulating geometries, which accelerated the stationary forward-backward method (FBM) [6] and decreased both the computational cost and memory requirements to $O(N)$. However, the spectrally accelerated FBM (SA-FBM) fails when the geometry of interest is a multivalued one, such as a reentrant surface of a ship, due to the nature of the FBM [7]. To overcome this problem, the spectrally accelerated generalized forward-backward method (SA-GFBM) and the multiblock generalized forward-backward method (MBGFBM) have been proposed in [8] and [9], respectively. It should be noted that in [8], although the overall computational cost is $O(N)$, the SA-GFBM becomes computationally expensive when the reentrant region becomes electrically large, whereas in [9], the proposed MBGFBM has an overall computational cost of $O\left(N^{2}\right)$ and is claimed to be reduced to $O(N)$ when combined with the SA algorithm. Naturally, the implementation of SA-GFBM and MBGFBM is scatterer specific, which is undesirable for the development of general purpose codes.

In this paper, electrically large rough-terrain profiles as well as reentrant geometries have been examined using the nonstationary iterative spectrally accelerated biconjugate gradient stabilized method (SA-BiCGSTAB) with a storage requirement and a computational cost of $O(N)$, where the SA principles given in [5] are used. Although the SA algorithm is valid for ordered surfaces (which becomes problematic for a reentrant geometry), such a geometry remains inside the strong field region in the SA-BiCGSTAB method, and requires a computational complexity of $O\left(N_{s}^{2}\right)$ (with $N_{s}$ the number of unknowns for the strong region) as opposed to $O\left(N_{s}^{3}\right)$, reported in [8]. Hence, in addition to its accuracy and robustness due to the nonstationary nature of BiCGSTAB method, the SA-BiCGSTAB method becomes more efficient than the SA-GFBM. Moreover, the $O\left(N_{s}^{2}\right)$ operational count for the strong region can be improved by using the fast-multipole method (FMM) in conjunction with the BiCGSTAB method [10]. Consequently, inherent robustness of a nonstationary technique (BiCGSTAB) has been merged with the SA algorithm in order to investigate electrically large arbitrary profiles efficiently and accurately. Such a hybrid method whose convergence characteristics is independent of the surface geometry, is very suitable for general scattering problems.

The paper is organized as follows: in section 2, the geometry and the formulation of the problem are given with a brief explanation of the spectrally accelerated BiCGSTAB method. In section 3 , numerical results composed of current distributions and path losses are given and compared with the measurements and previously published results in order to assess the accuracy and efficiency of the method. An $e^{j \omega t}$ time convention is employed and suppressed throughout the paper. $\omega, k$, and $\lambda$ are the angular frequency, wave number, and wavelength of free space, respectively.

\section{FORMULATION}

An arbitrary-terrain profile with no variation along the transverse direction of the propagating field, as in [4-9, 11-14] is depicted in Figure 1. The electromagnetic fields characterized by $\boldsymbol{E}^{i}$ and $\boldsymbol{H}^{i}$ are incident upon the surface. The terrain profile is modeled to be an imperfect conductor (with permeability $\mu$ and permittivity $\epsilon$ ) and analyzed using an impedance boundary condition (IBC) [15] to be able to investigate more general situations.

\subsection{IE and MoM Formulation}

An IE is formed by applying IBCs on the surface of the scatterer, and the EFIE for a TM wave can be written as

$$
\begin{aligned}
-E_{y}^{i}=-\eta_{s} J_{y}(\rho)-\frac{\omega \mu}{4} & \int_{C} J_{y}\left(\rho^{\prime}\right) H_{0}^{(2)}\left(k\left|\rho-\rho^{\prime}\right|\right) d \rho^{\prime} \\
& \quad-j \frac{k \eta_{s}}{4} \int_{C} J_{y}\left(\rho^{\prime}\right) \hat{n} \cdot \hat{\rho} H_{1}^{(2)}\left(k\left|\rho-\rho^{\prime}\right|\right) d \rho^{\prime} .
\end{aligned}
$$




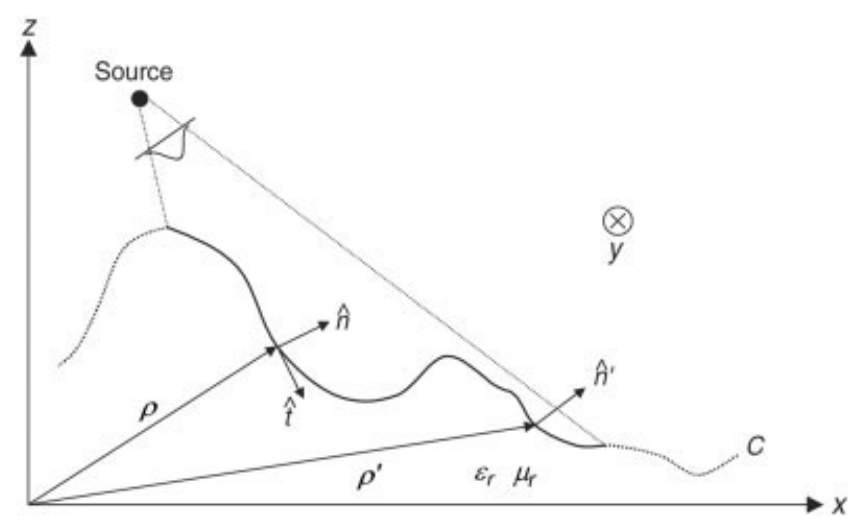

Figure 1 An arbitrary terrain profile

In Eq. (1), $E_{y}^{i}$ is the incident field, $J_{y}$ is the unknown current distribution to be solved, which is tangential in the transverse direction to the plane of the surface, $\eta_{s}$ is the surface impedance of the structure, $\rho$ and $\rho^{\prime}$ are the observation and source points, respectively, $\hat{n}$ is the unit-normal vector at the source point, $C$ is the contour of the surface and, lastly, $H_{0}^{(2)}$ and $H_{1}^{(2)}$ are the Hankel functions of the second kind with orders of zero and one, respectively. Similar to the TM case, the MFIE is utilized for the TE polarization by assuming that a TE magnetic source is incident upon the surface, and can be expressed as

$$
\begin{aligned}
-H_{y}^{i}=J_{t}(\rho)+\frac{\omega \epsilon \eta_{s}}{4} \int_{C} J_{t}\left(\rho^{\prime}\right) H_{0}^{(2)}\left(k\left|\rho-\rho^{\prime}\right|\right) d \rho^{\prime} & \\
& \quad+j \frac{k}{4} \int_{C} J_{t}\left(\rho^{\prime}\right) \hat{n} \cdot \hat{\rho} H_{1}^{(2)}\left(k\left|\rho-\rho^{\prime}\right|\right) d \rho^{\prime} .
\end{aligned}
$$

In Eq. (2), $H_{y}^{i}$ is the incident field and $J_{t}$ is the unknown current distribution to be solved, which is tangential to the surface. Although the MFIE can be applied only to closed surfaces, the surface in this problem is assumed to extend to infinity, since the source illuminates only a limited region on the surface. Expanding the unknown surface current density $J\left(\rho^{\prime}\right)$, whether $J_{y}$ in Eq. (1) or $J_{t}$ in Eq. (2), by $N$ (the number of unknowns) unit pulse basis functions and using a point matching technique, the following matrix equation is formed:

$$
\overline{\mathbf{Z}} \cdot \mathbf{I}=\mathbf{V}
$$

In Eq. (3), $\mathbf{V}$ is the excitation vector which contains the minus of the incident field at the observation points $\left(-E_{y}^{i}(\rho)\right.$ in (1) or $-H_{y}^{i}(\rho)$ in (2)), $\mathbf{I}$ has the unknown current coefficients, and $\overline{\mathbf{Z}}$ is the impedance matrix whose entries are given by

$$
Z_{n m} \cong \begin{cases}-\frac{\omega \mu}{4}\left[1-j \frac{2}{\pi} \ln \left(\frac{\gamma k \Delta x_{m}}{4 e}\right)\right] \Delta x_{m}-\frac{\eta_{m}}{2} & n=m \\ -j \omega \mu G\left(\rho_{n}, \rho_{m}\right) \Delta x_{m}+\eta_{m} \Delta x_{m} \frac{\partial G\left(\rho_{n}, \rho_{m}\right)}{\partial n_{m}} & n \neq m\end{cases}
$$

for the MFIE (TM polarization case) and

$$
Z_{n m} \cong \begin{cases}\frac{\omega \epsilon \eta_{m}}{4}\left[1-j \frac{2}{\pi} \ln \left(\frac{\gamma k \Delta x_{m}}{4 e}\right)\right] \Delta x_{m}+\frac{1}{2} & n=m \\ j \omega \epsilon \eta_{m} G\left(\rho_{n}, \rho_{m}\right) \Delta x_{m}-\Delta x_{m} \frac{\partial G\left(\rho_{n}, \rho_{m}\right)}{\partial n_{m}} & n \neq m\end{cases}
$$

for the MFIE (TE polarization case). $G\left(\rho_{n}, \rho_{m}\right)$ in Eqs. (4) and (5) is the $2 \mathrm{D}$ Green's function, which can be expressed by

$$
G\left(\rho_{n}, \rho_{m}\right)=\frac{H_{0}^{(2)}\left(k\left|\rho_{n}-\rho_{m}\right|\right)}{4 j} .
$$

Once the impedance matrices [whose entries are given in (4) and (5) for TM and TE polarizations, respectively] are formed, the corresponding matrix equation given in (3) should be solved for the unknown current coefficients $\mathbf{I}=\left\{I_{m}\right\}$.

\subsection{Spectrally Accelerated Biconjugate Gradient Stabilized Method}

The BiCGSTAB method is a nonstationary iterative method whose pseudo code can be found in [16]. The storage and computational costs (due to two matrix-vector multiplies at each iteration) of the method $\left(O\left(N^{2}\right)\right)$ are improved to $O(N)$ using the SA algorithm, which is mainly based on a spectral-domain representation of the 2D Green's function. The SA algorithm is applicable to both PEC and imperfect-conducting cases, and although it is well suited for the FBM in that it precedes the forward and backward sweeps of the propagating fields, it can be used in any standard iterative technique as well, since these sweeps are represented by matrixvector multiplies. Here, only the basic concepts of the SA algorithm are given. Interested readers may refer to $[4,5]$ for more details.

In the SA algorithm, radiating elements over a given receiving element are divided into two groups: namely, one is the forwardpropagating field ( $E_{f}$ for the EFIE, $H_{f}$ for the MFIE) via preceding source elements including the source element itself, and the backward-propagating field ( $E_{b}$ for the EFIE, $H_{b}$ for the MFIE) via the following source elements with respect to the $n^{\text {th }}$ receiving point. The forward-propagating field for $E_{f}$ for the TM polarization (the same assumption is valid for the TE polarization case) is also decomposed into two groups: the strong interaction group and the weak interaction group, which are expressed as

$$
E_{f}\left(\rho_{n}\right)=E_{f, s}\left(\rho_{n}\right)+E_{f, w}\left(\rho_{n}\right)
$$

with

$$
E_{f, s}\left(\rho_{n}\right)=\sum_{m=n-N_{s}+1}^{n} I_{m} Z_{n m}
$$

and

$$
E_{f, w}\left(\rho_{n}\right)=\sum_{m=n-N_{s}+1}^{n-N_{s}} I_{m} Z_{n m} .
$$

The decisive factor defining these groups is the distance of a radiating element from the receiving element. Thus, for a distance $L_{s}$ (which is very small compared to the length of the terrain), the strong group contains $N_{s}=L_{s} / \Delta x$ (with $\Delta x$ the unit-pulse width) elements $\left(N_{s} \ll N\right.$, independent of the maximum height deviation and the number of unknowns) including the self interaction, whereas the rest of the source elements construct the weak group. Radiations from the strong group are found by the matrix-vector multiplication of exact impedance elements yielding $O\left(N_{s}^{2}\right)$ operations. However, the weak-group contribution is obtained by employing the spectral representation of the 2D Green's function, given by 


$$
G\left(\rho_{n}, \rho_{m}\right)=\frac{-j}{4 \pi} \int_{C_{\phi}} e^{-j k\left[\left(x_{n}-x_{m}\right) \cos \phi+\left(z_{n}-z_{m}\right) \sin \phi\right]} d \phi,
$$

where $C_{\phi}$ is the contour of integration in the complex angular space. The derivative of the Green's function (which takes place within the off-diagonal entries of the impedance matrix) can be found by taking the derivative of (10) with respect of the normal vector at the source point. Substituting (10) and its derivative into (9), one can derive the forward weak field as an integral in the complex $\phi$ space, whose integrand can be given by the following recursive relation:

$$
\begin{aligned}
F_{n}(\phi) & =F_{n-1}(\phi) e^{\left.-j k\left[\left(x_{n}-x_{n-1}\right) \cos \phi+\left(z_{n}-z_{n-1}\right) \sin \phi\right)\right]}+I_{n s} \Delta x_{n s}\{1 \\
- & \frac{\eta_{n s}}{\eta_{0}}\left[\cos \theta_{n s} \cos \phi+\sin \theta_{n, s} \sin \phi\right] e^{-j k\left[\left(x_{n}-x_{n s}\right) \cos \phi-\left(z_{n}-z_{n s}\right) \sin \phi\right]},
\end{aligned}
$$

where $n s=n-N_{s}$. Due to the recursive procedure in (11), the radiated field from the $n^{\text {th }}$ element updates itself in terms of the previous one. Hence, the computational cost required for the weak region is $O\left(N-N_{s}\right)$. The final field value for the forward field is the sum of the strong and weak region contributions. Since $(N-$ $\left.N_{s}\right) \gg N_{s}$, a total operational cost of $O(N)$ is maintained. Note that details related to the efficient evaluation of the complex $\phi$ integral which yields the forward weak field can be found in [5].

The recursive relation in (11) and the deformation of the integration contour are valid for ordered surfaces which create problems if a reentrant geometry is analyzed. By including this reentrant geometry into the strong field region in the SA-BiCGSTAB method, the operation count of the strong region is $O\left(N_{s}^{2}\right)$, which is more efficient than $O\left(N_{s}^{3}\right)$ in the SA-GFBM. A similar procedure is used for backward propagation.

\section{NUMERICAL RESULTS}

To assess the accuracy and efficiency of the SA-BiCGSTAB method, several numerical examples, in the form of induced current and path loss, are obtained and compared with the results in previously published papers, as well as the measurements. The

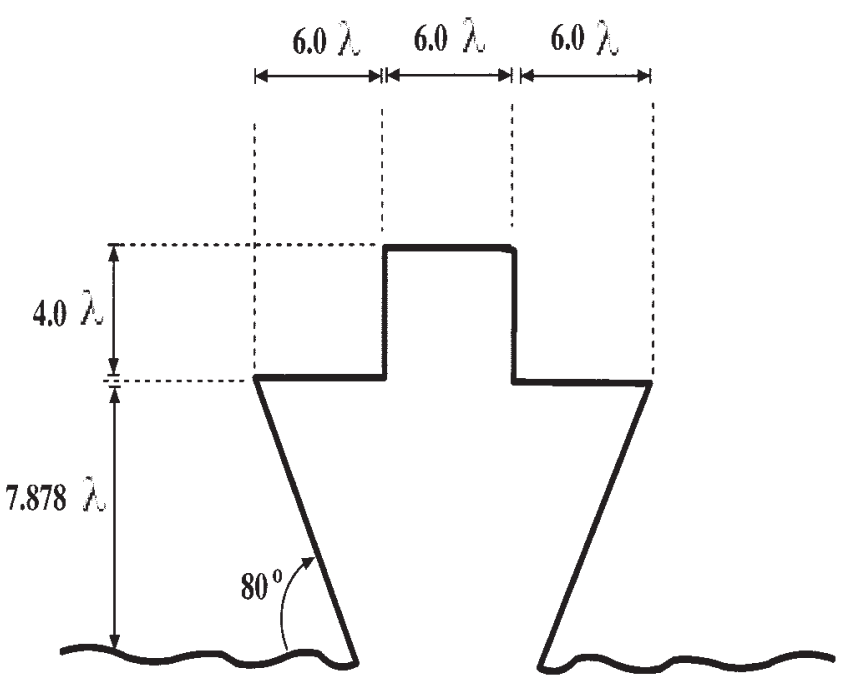

Figure 2 Ship geometry on the sea (with respect to $\lambda$ )
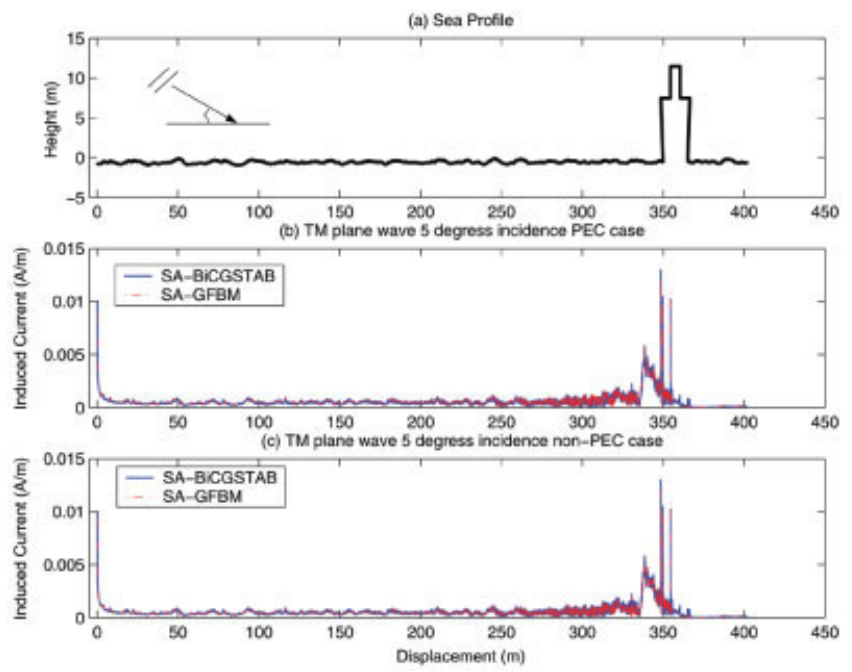

Figure 3 Current distribution on the sea and the ship at $300 \mathrm{MHz}$ (wind speed $=5 \mathrm{~m} / \mathrm{s}$ ) with $N \simeq 4500$ and no. of iterations $=69$. [Color figure can be viewed in the online issue, which is available at www. interscience.wiley.com]

stopping criteria of the SA-BiCGSTAB method is to reduce the residual error below a level of $10^{-3}$.

The first example is an unordered surface profile such that the target under test is a ship placed on a $400-\mathrm{m}$ sea surface. The dimensions of the ship in terms of $\lambda$ are shown in Figure 2. Random sea-surface specifications can be found in [8]. The results are demonstrated in the form of current distributions for a TM polarized plane wave with a $5^{\circ}$ incident angle. The profiles of the sea and the ship at $5-\mathrm{m} / \mathrm{s}$ and $10-\mathrm{m} / \mathrm{s}$ wind speed are depicted in Figures 3(a) and 4(a), respectively. Figures 3(b) and 4(b) show the results when both the sea and the ship are considered to be PEC. In Figures 3(c) and 4(c), the surface impedance of the sea is taken as $\eta_{s}=76.4+j 65.1$ while the ship remains PEC. As can be seen in both figures, the agreement of the SA-BiCGSTAB method with the SA-GFBM is very good. The frequency is $300 \mathrm{MHz}$, yielding approximately 4500 unknowns. The total number of iterations is
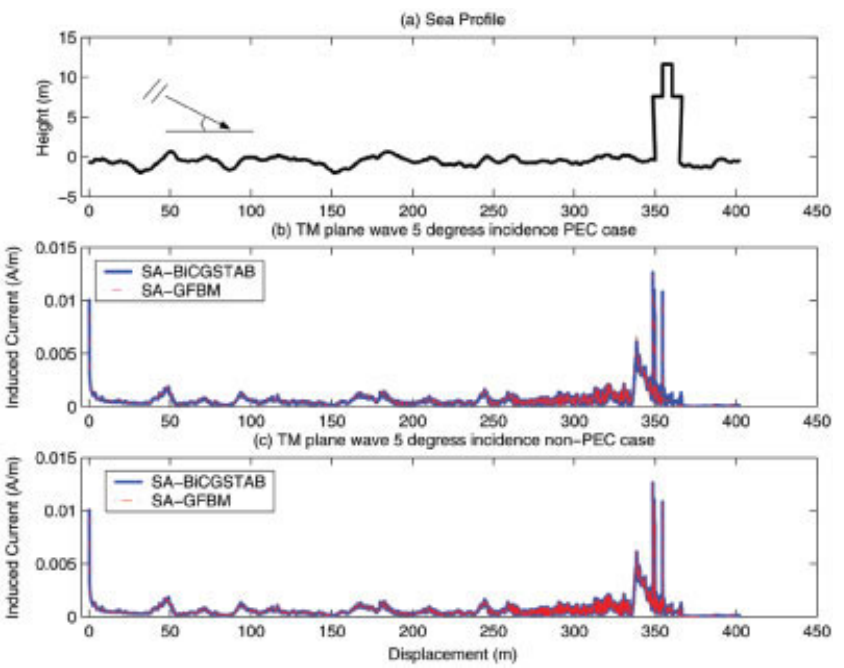

Figure 4 Current distribution on the sea and the ship at $300 \mathrm{MHz}$ (wind speed $=10 \mathrm{~m} / \mathrm{s}$ ) with $N \simeq 4500$ and no. of iterations $=69$. [Color figure can be viewed in the online issue, which is available at www. interscience.wiley.com] 


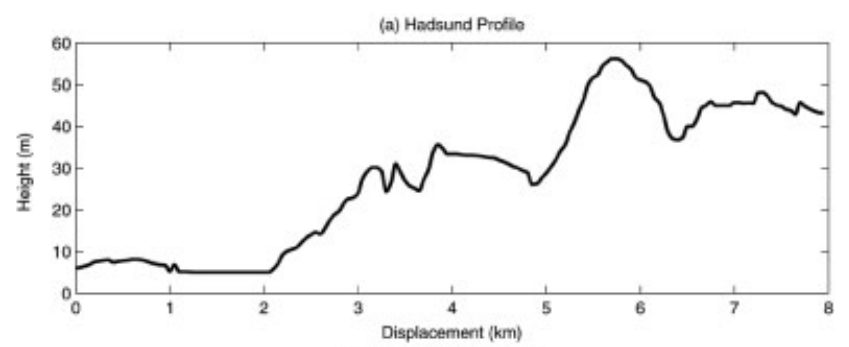

(b) PATH LOSS at $435 \mathrm{MHz}$

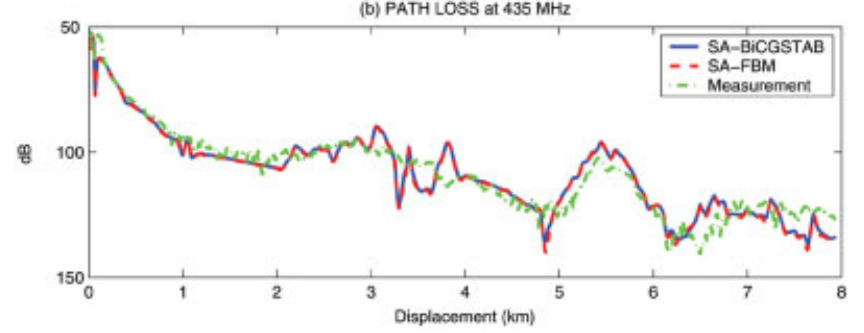

Figure 5 Hadsund terrain-profile measurements at $435 \mathrm{MHz}$ compared with the SA-BiCGSTAB method and the SA-FBM with $N=115274$ and no. of iterations $=32$ (for SA-BiCGSTAB) and 7 (for SA-FBM). [Color figure can be viewed in the online issue, which is available at www. interscience.wiley.com]

69. In this example, although the number of the iterations for the SA-BiCGSTAB method is larger than that of SA-GFBM [8], for geometries with electrically large reentrant parts (such as multiships), SA-BiCGSTAB method becomes advantageous.

The next set of numerical examples are related to the investigation of scattering from electrically large terrain profiles. The analyses have been done on real terrain profiles which take place in Northern Jutland in Denmark. To show the accuracy of the method, the results are compared with measurements at different frequencies, which were performed by Hviid et al. [12]. Two profiles with a path length of $6-8 \mathrm{~km}$ and height deviation of 20-50 $\mathrm{m}$ are examined. The TM polarization case is considered. Since no information is received about the plant cover or the electrical properties of the terrains, the surface impedance is taken as $\eta_{s}=20+j 8.2$ to simulate spinneylike land cover. The source is considered to be a dipole antenna located at the left-most point of the terrain above a height of $10 \mathrm{~m}$ with a transmit power of 10 $\mathrm{W}$ and gain of $8 \mathrm{dBi}$. The scattered field is evaluated at $2.4 \mathrm{~m}$ above the terrain by using the SA once again resulting $O(N)$ operations. The results are also compared with the SA-FBM [17] in order to demonstrate that the path-loss values obtained using another numerical technique perfectly match the values determined by the SA-BiCGSTAB method, when the electrical properties of the terrain and the source are the same.

The Hadsund measurements and the results of the SA-BiCGSTAB method are plotted in Figure 5 at a frequency of $435 \mathrm{MHz}$. The prediction of the SA-BiCGSTAB smoothly fits the curve of the measurement data. Furthermore, the field values found by the SA-BiCGSTAB method and the SA-FBM perfectly match each other. A similar result is obtained for the Jerslev terrain, which is depicted in Figure 6 at a frequency of $970 \mathrm{MHz}$. As illustrated in Figure 6, the agreement of the SA-BiCGSTAB method with the measurement results, as well as with the SA-FBM, is very good.

Path-loss examples show that from the broadcasting point of view, the SA-BiCGSTAB method is very accurate for use in the propagation analyses. Furthermore, the excellent agreement between the SA-FBM and SA-BiCGSTAB methods also shows that the SA-BiCGSTAB method, as it is a nonstationary numerical
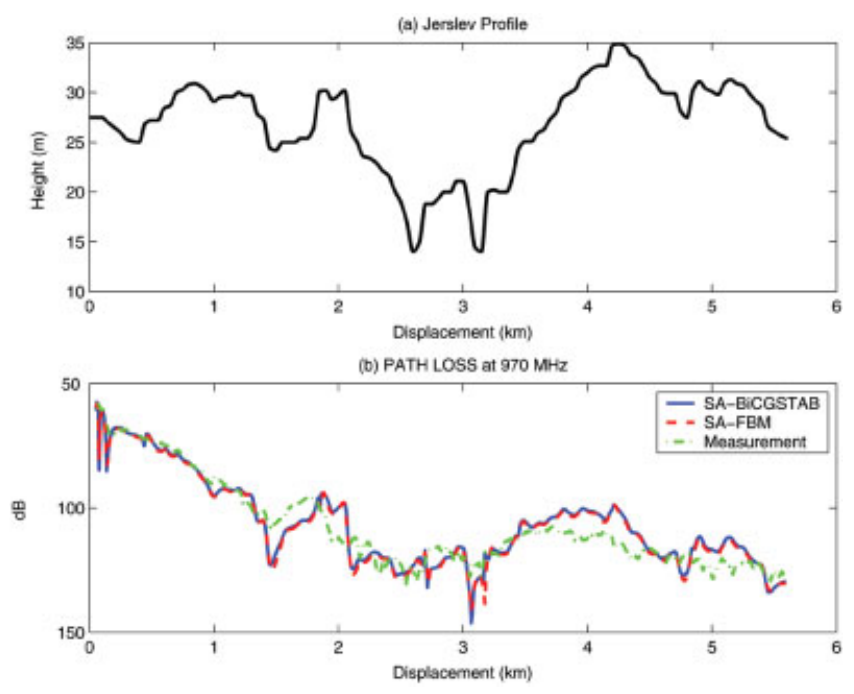

Figure 6 Jerslev terrain profile measurements at $970 \mathrm{MHz}$ compared with the SA-BiCGSTAB method and the SA-FBM with $N=191018$ and no. of iterations $=32$ (for SA-BiCGSTAB) and 7 (for SA-FBM). [Color figure can be viewed in the online issue, which is available at www. interscience.wiley.com]

technique, can be used for electrically large terrain-scattering problems with or without an electrically large reentrant part.

\section{CONCLUSION}

Scattering and propagation analysis over rough surface profiles as well as reentrant geometries have been examined using an IE, which is solved by the SA-BiCGSTAB method with a storage and computational cost of $O(N)$ per iteration. The results were presented in the form of current distributions and path losses and were compared with previously published results and measurements in order to assess the accuracy of the method. Due to the nonstationary nature of the BiCGSTAB method, precise results can be obtained even for reentrant structures without any computational complexity, which cannot be handled by any stationary methods. Apart from that, the method is also applicable for MFIE problems with more rapid convergence characteristics due to better conditioning of the system interactions. By merging the robust characteristics of nonstationary techniques with the SA algorithm, the SA-BiCGSTAB method can be employed for general scattering problems.

\section{ACKNOWLEDGMENTS}

The authors would like to thank Dr. M. R. Pino and his associates for kindly supplying the ship geometries and the results of the spectrally accelerated generalized forward-backward method [8]. The authors also thank Prof. J. B. Andersen and his associates for kindly providing the terrain profiles and integral equation method results [12].

\section{REFERENCES}

1. Y. Okumura, E. Ohmori, T. Kawano, and K. Fukuda, Field strength and its variability in VHF and UHF lan-mobile radio service, IEEE Trans Antennas Propagat 16 (1968), 825-873.

2. M. Hata, Empirical formula for propagation loss on land mobile radio services, IEEE Trans Vechic Technol 33 (1985), 1045-1053.

3. VHF and UHF propagation curves for the frequency range from 30 MHz to 1000 MHz, Rec. ITU-R P. 370-7, ITU, 1995.

4. H.-T. Chou and J.T. Johnson, A novel acceleration for the computation 
of scattering from rough surfaces with the forward-backward method, Radio Sci 33 (1998), 1277-1287.

5. J.A. Lopez, M.R. Pino, F. Obelleiro, and J.L. Rodriguez, Application of the spectral acceleration forward-backward method to coverage analysis over terrain profiles, J Electromagn Waves Appl 15 (2001), 1049-1074.

6. D. Holliday, L.L. DeRaad, Jr., and G.J. St.-Cyr, Forward-backward: A new method for computing low-grazing scattering, IEEE Trans Antennas Propagat 44 (1996), 722-729.

7. J.C. West, On iterative approaches for electromagnetic rough-surface scattering problems, IEEE Trans Antennas Propagat 47 (1999), 12811288.

8. M.R. Pino, R.J. Burkholder, and F. Obelleiro, Spectral acceleration of the generalized forward-backward method, IEEE Trans Antennas Propagat 50 (2002).

9. M.R. Pino, F. Obellerio, and J.L. Rodriguez, A multiblock generalized forward-backward method, Radio Sci 36 (2001), 19-29.

10. O.S. Ergul, Fast multipole method for the solution of electromagnetic scattering problems, M. Eng. Thesis, Bilkent University, Ankara, Turkey, June 2003.

11. R. Janaswamy, A Fredholm integral equation method for propagation over small terrain irregularities, IEEE Trans Antennas Propagat 40 (1994), 1416-1422.

12. J.T. Hviid, J.B. Anderson, J. Toftgard, and J. Bojer, Terrain based propagation model for rural area - an integral equations approach, IEEE Trans Antennas Propagat 43 (1995), 41-46.

13. J.T. Johnson, R.T. Shin, J.C. Eidson, L. Tsang, and J.A. Kong, A method of moments model for VHF propagation, IEEE Trans Antennas Propagat 45 (1997), 115-125.

14. E.O. Nuallain, An improved method to evaluate the slow fading signal over electrically massive scatterers, in IEEE Inter Antennas Propagat Sym and USNC/URSI Nat Radio Sci Meeting, 20-25 June 2004, California, USA.

15. L.N. Medgyesi-Mitschang and J.M. Putnam, Integral equation formulations for imperfectly conducting scatterers, IEEE Trans Antennas Propagat AP-33 (1985).

16. R. Barret, M. Berry, T.F. Chan, J. Demmel, J. Donato, J. Dongarra, V. Eijkhout, R. Poza, C. Romine, and H. Ban der Vort, Templates for the solution of linear systems: building blocks for iterative methods, Philadelphia, PA: SIAM, 1994.

17. C.A. Tunc, A. Altintas, and V.B. Ertürk, Propagation and coverage analysis over terrain profiles comparing empirical approaches with exact solutions, in IEEE Inter Antennas Propagat Sym and USNC/ CNC/URSI North Am Radio Sci Meeting, June 2003, Columbus, Ohio, USA.

(C) 2005 Wiley Periodicals, Inc.

\section{MULTI-WAVELENGTH ERBIUM-DOPED FIBER LASER BASED ON A MICROSTRUCTURE FIBER BRAGG GRATING}

\author{
Tingting Sun, Guiyun Kai, Zhi Wang, Chao Wang, \\ Chunshu Zhang, Yange Liu, Jianfei Liu, Weigang Zhang, \\ Shuzhong Yuan, and Xiaoyi Dong \\ Institute of Modern Optics \\ Nankai University \\ Tianjin, P. R. China
}

Received 13 December 2004

ABSTRACT: In this paper, a multiwavelength erbium-doped fiber laser based on a microstructure fiber Bragg grating $(F B G)$ is proposed and demonstrated. The fiber Bragg grating is fabricated in a large-air-hole microstructure optical fiber using the phase-mask method. The laser based on this novel grating can be designed to achieve a three-wavelength output at room temperature. The lasing wavelengths of the channels are 1557.84, 1555.07, and $1552.70 \mathrm{~nm}$, respectively, and the wavelength separation is about $2 \mathrm{~nm}$. (C) 2005 Wiley Periodicals, Inc. Microwave Opt Technol Lett 46: 162-164, 2005; Published online in Wiley InterScience (www.interscience.wiley.com). DOI 10.1002/mop. 20931

Key words: erbium-doped fiber laser (EDFL); multiwavelength fiber laser; microstructure fiber $(M F)$; fiber Bragg grating ( $F B G)$

\section{INTRODUCTION}

Multiwavelength fiber lasers are useful sources in wavelengthdivision-multiplexed (WDM) fiber-communication systems, fiber sensors, and optical-instrument testing. With regard to wavelength-selection components for lasers, fiber Bragg gratings (FBGs) are ideal ones due to the unique advantage of fiber compatibility. Various techniques have been proposed to realize multiwavelength oscillations by utilizing cascaded FBG cavities [1], polarization-dependent loss element [2], an FBG written in a birefringent fiber [3, 4], a sampled FBG [5], two overlapping cavities composed of two FBGs with a common gain medium [6], and FBG fabricated in a few-mode fiber [7].

Air-silica microstructure fibers are all-silica fibers which contain air voids that run along the length of the fiber. Such novel optical fibers differ from traditional fibers with regard to their optical properties, due to the effect of the structure of the fiber cladding on the spatial distribution and the effective refractive indices of cladding mode, thus increasingly attracting attention to this class of fiber-waveguide structures. Recently, increased interest has focused on the guidance properties of the cladding modes. The inscription of both FBG and long-period grating in microstructure fibers with different geometrical cross sections has been reported [8-11]. In this paper we choose a "grapefruit" fiber, which is a typical large-air-hole microstructure fiber, for fabrication of an FBG in the photosensitive core using the phase-mask method. The grating formed in this novel waveguide shows multiple resonances in both its transmission and reflection spectra, as compared to conventional FBGs in single-mode fibers. This FBG is incorporated into an Erbium-doped fiber-laser cavity as a wavelength-selective component for the first time, to the best of our knowledge. The proposed laser can be made to operate with a three-wavelength output at room temperature. The lasing wavelengths of the oscillations are $1557.84,1555.07$, and $1552.70 \mathrm{~nm}$, respectively, and the wavelength separation is about $2 \mathrm{~nm}$. The output powers for the three wavelengths are $-5.2,-3.1$, and -4.1 $\mathrm{dBm}$, respectively. 